ThE Astrophysical Journal, 469:L1-L4, 1996 September 20

(C) 1996. The American Astronomical Society. All rights reserved. Printed in U.S.A.

\title{
DISCOVERY OF SUBMILLISECOND OUASI-PERIODIC OSCILLATIONS IN THE X-RAY FLUX OF SCORPIUS X-1
}

\author{
M. VAN DER KLIS \\ Astronomical Institute “Anton Pannekoek," University of Amsterdam, and Center for High-Energy Astrophysics, Kruislaan 403, NL-1098 SJ Amsterdam, \\ The Netherlands \\ J. H. SWANK, W. ZHANG, AND K. JAHODA \\ Goddard Space Flight Center, NASA, Greenbelt, MD 20771 \\ E. H. Morgan AND W. H. G. Lewin \\ Massachusetts Institute of Technology, Cambridge, MA 02139 \\ B. VAUGHAN \\ California Institute of Technology, Pasadena, CA 91125 \\ AND \\ J. VAN PARADIJS \\ Department of Physics, University of Alabama at Huntsville, Huntsville, AL 35899, and University of Amsterdam \\ Received 1996 April 25; accepted 1996 July 5
}

\begin{abstract}
We report the discovery, with NASA's Rossi X-Ray Timing Explorer (RXTE), of the first submillisecond oscillations found in a celestial X-ray source. The quasi-periodic oscillations (QPOs) come from Sco X-1 and have a frequency of $\sim 1100 \mathrm{~Hz}$ and amplitudes of $0.6 \%-1.2 \%(\mathrm{rms})$ and are relatively coherent, with $Q$ up to $\sim 10^{2}$. The frequency of the QPOs increases with accretion rate, rising from 1050 to $1130 \mathrm{~Hz}$ when the source moves from top to bottom along the normal branch in the X-ray color-color diagram, and shows a strong, approximately linear correlation with the frequency of the well-known 6-20 Hz normal/flaring-branch QPOs. We also report the discovery of QPOs with a frequency near $800 \mathrm{~Hz}$ that occur, simultaneously with the $1100 \mathrm{~Hz}$ QPOs, in the upper normal branch. We discuss several possible interpretations, one involving a millisecond X-ray pulsar whose pulses we see reflected off accretion flow inhomogeneities. Finally, we report the discovery of $\sim 45 \mathrm{~Hz}$ QPOs, most prominent in the middle of the normal branch, which might be magnetospheric beat-frequency QPOs.

Subject headings: pulsars: general — stars: individual (Scorpius X-1) — stars: neutron
\end{abstract}

\section{INTRODUCTION}

The characteristic timescale for motion of matter near a gravitating object, the dynamical timescale $\tau_{\mathrm{dyn}}$, is $\left(r^{3} / G M\right)^{1 / 2}$, where $r$ is the distance to the object and $M$ is its mass. For $r=R$, the size of the object, $\tau_{\mathrm{dyn}}$ is relevant to possible spin and vibration periods. For a neutron star, $\tau_{\mathrm{dyn}}$ extends to values of well below $1 \mathrm{~ms}$. Submillisecond variability could therefore be produced in an accreting neutron star by various mechanisms. In this Letter, we present the first conclusive evidence for submillisecond variability in an accreting neutron star, Sco X-1. A preliminary announcement of this work was already made in van der Klis et al. (1996).

Sco X-1 (see Giacconi et al. 1962), the brightest persistent $\mathrm{X}$-ray source in the sky, is a $\mathrm{Z}$ source (Hasinger \& van der Klis 1989), a luminous low-mass X-ray binary containing a low magnetic field neutron star. In the X-ray color-color diagram, it shows the lower two of the three branches in the canonical "Z track," the normal branch (NB) and the flaring branch (FB) (Middleditch \& Priedhorsky 1986; Priedhorsky et al. 1986; Hertz et al. 1992; Dieters \& van der Klis 1996). Its power spectrum is characterized by the presence of $1 \%-5 \%$ amplitude, $6-20 \mathrm{~Hz}$ normal/flaring-branch quasi-periodic oscillations (N/FBOs). According to the standard interpretation (see, e.g., Hasinger et al. 1990; Lamb 1991), when the mass transfer rate $\dot{M}$ to the neutron star increases, the source moves down along the NB, passes through the "vertex" of the two branches, where it reaches the Eddington critical rate, and then moves up the FB. At the same time, the quasi-periodic oscillation (QPO) frequency increases from $\sim 6 \mathrm{~Hz}$ on the NB to $\sim 20 \mathrm{~Hz}$ on the lower FB. Further up the FB, the QPOs disappear. Below we report three new, previously unknown QPO phenomena in this source and describe their dependence on $\dot{M}$.

\section{OBSERVATIONS}

Sco $\mathrm{X}$-1 was observed three times with the proportional counter array (PCA) on board NASA's Rossi X-Ray Timing Explorer (RXTE; see Bradt, Rothschild, \& Swank 1993), on 1996 February 14 from 0914 to 1325 UT, February 180446 0840 UT, and February 19 1009-1456 UT, hereafter observations 1, 2, and 3, respectively. Each observation covered three satellite orbits with $\sim 2500 \mathrm{~s}$ of data, separated by intervals of $\sim 1500 \mathrm{~s}$ due to Earth occultation, South Atlantic Anomaly passage, or both. The count rate was $\sim 10^{5}$ counts $\mathrm{s}^{-1}$ in observations 1 and 2 . At the start of orbit 1 of observation 3 , the count rate increased to $\sim 1.45 \times 10^{5}$ counts $\mathrm{s}^{-1}$, which tripped the PCA high-voltage safety switch-off. RXTE was then moved slightly off Sco X-1 to $\sim 50 \%$ collimator efficiency; later count rates were between $0.5 \times 10^{5}$ and $0.8 \times 10^{5}$ counts $\mathrm{s}^{-1}$. The background below $20 \mathrm{keV}$ was $\lesssim 90$ counts s${ }^{-1}$ and has been neglected.

During orbits 1 and 3 of each observation, data were collected with a time resolution of initially $0.5 \mathrm{~ms}$ and later, after discovery of the $1100 \mathrm{~Hz}$ QPOs in observation 1, usually 


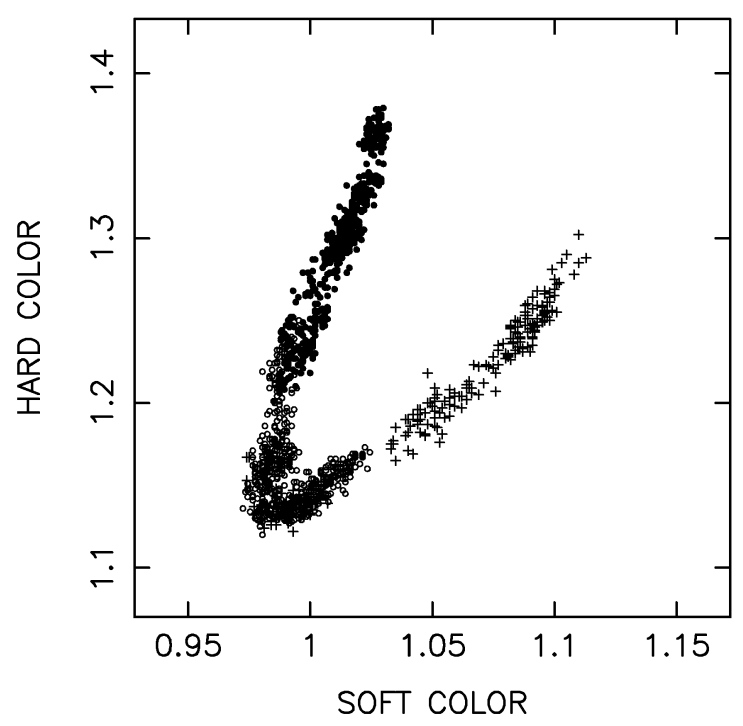

Fig. 1.-X-ray color-color diagram. Soft color is the (3-5 keV)/(1-3 keV), hard color the $(7-20 \mathrm{keV}) /(5-7 \mathrm{keV})$, count-rate ratio. Each data point corresponds to $16 \mathrm{~s}$ of data; filled circles, open circles, and plus signs correspond to observations 1,2 , and 3 , respectively. The statistical uncertainties are $\sim 0.003$ in soft color and $\sim 0.005$ in hard color. The systematic uncertainties in Z-track location between observations (see § 3) are $\sim 1 \%$.

$0.25 \mathrm{~ms}$. During orbit 2 of each observation, the time resolution was set to $16 \mu \mathrm{s}$, and "double events" were additionally recorded at $64 \mu \mathrm{s}$ time resolution. Double events, two events detected within $6 \mu \mathrm{s}$ at two different anodes of a PCA detector, are normally mostly due to charged particles. However, for the very high count rates from Sco X-1, they are mostly due to two source photons. In the data analysis, the double events, counted as two, were added to the singlephoton data. This led to a considerable increase in sensitivity to time variability. All high time resolution data were recorded in the $2-20 \mathrm{keV}$ band. During the entire run, $16 \mathrm{~s}$ resolution data were additionally recorded in 129 spectral channels covering the $2-60 \mathrm{keV}$ band.

\section{ANALYSIS AND RESULTS}

We used the $16 \mathrm{~s}$ data to construct X-ray color-color diagrams. There was a considerable offset between the $\mathrm{Z}$ track seen in observations 1 and 2 and that in observation 3 , which we attribute to energy-dependent detector effects (collimator reflections, rate-dependent gains, or both). After an empirical correction that assumes that the $\mathrm{Z}$ track did not move between our observations (see, e.g., Dieters \& van der Klis 1996), a typical Sco X-1 color-color diagram results (Fig. 1). During observation 1 , the source moved gradually down the NB; during observation 2 , it moved up and down between the lower $\mathrm{NB}$ and FB; and in observation 3, it moved gradually down the FB and into the vertex region. We assigned numbers $S_{Z}$ to positions on the $\mathrm{Z}$ track according to curve length along the track, where we set the top of our NB to 1 and the vertex to 2 .

Approximately $1100 \mathrm{~Hz}$ QPOs (Fig. 2) were observed on all occasions that the source was on the NB or very low on the FB and the observational setup was adequate (full collimator efficiency to ensure sufficient sensitivity, and sufficient time resolution). This was the case in orbit 2 of observation 1 and in orbits 1 and 2 of observation 2. The QPOs were seen independently in single- and double-event data. Table 1 lists the results of functional fits of a constant plus a power law plus one or two Lorentzian peaks to power spectra of the high time

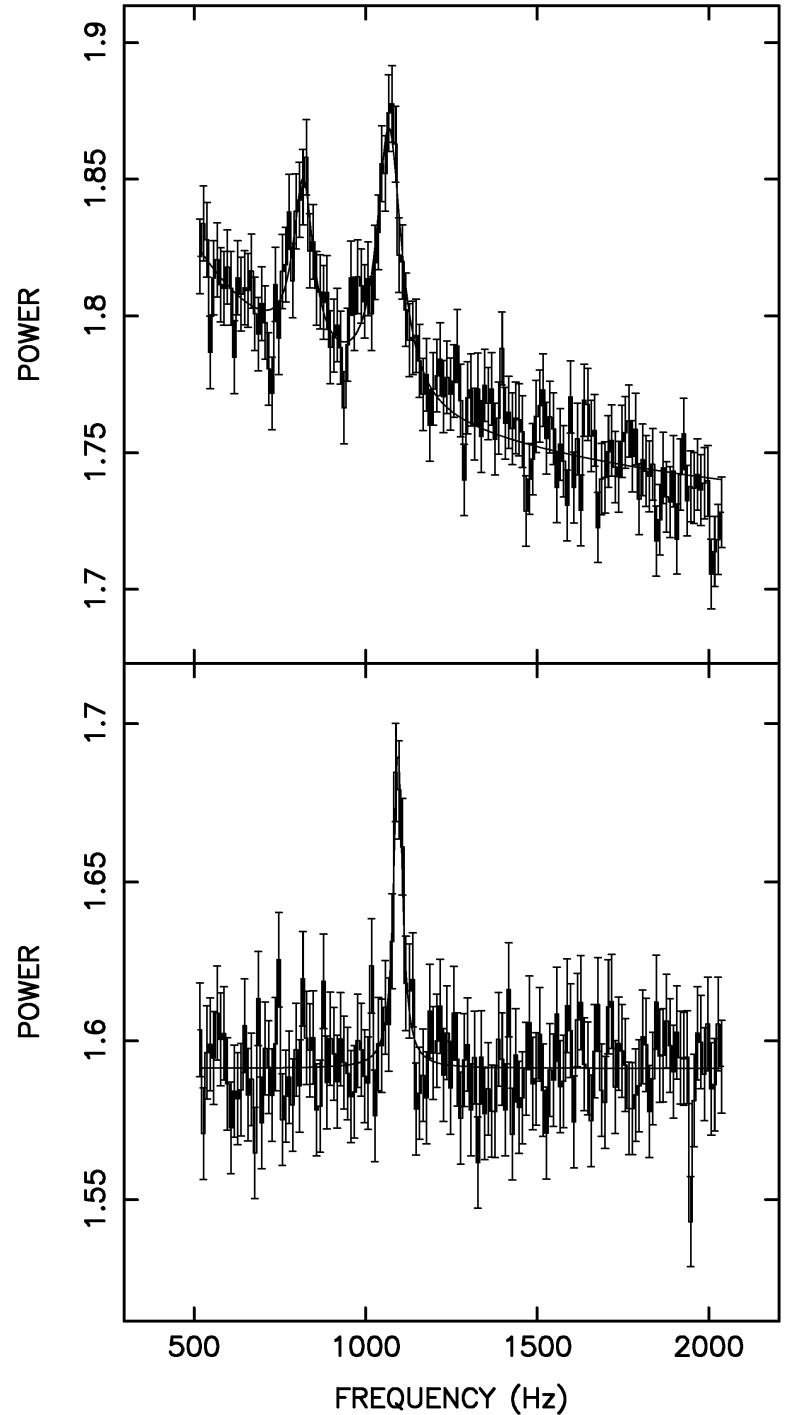

FIG. 2.-Power spectra of combined single- and double-event data (see § 2) showing simultaneous 800 and $1100 \mathrm{~Hz}$ QPOs (top; orbit 2 of observation 1) and a narrow $1100 \mathrm{~Hz}$ QPO peak (bottom; orbit 2 of observation 2). The spectra are Leahy-normalized; the offsets of the continua from a level of 2.0 and the slope of the continuum in observation 1 are due to instrumental dead-time effects.

resolution data. All fits were statistically acceptable, with reduced $\chi^{2} \sim 1$. The QPO frequency $\nu_{1100}$ increased with $\dot{M}$, from $1050 \mathrm{~Hz}$ at $S_{\mathrm{Z}}=1.25$ to $1130 \mathrm{~Hz}$ at $S_{\mathrm{Z}}=2.1$. Note that, for lack of time resolution, we have no information about the $1100 \mathrm{~Hz}$ QPOs in the $S_{Z}$ range 1.4-1.8. The QPOs were sometimes quite coherent, with $Q \equiv \nu / \Delta \nu$ up to $10^{2}$. Fractional rms amplitudes, corrected for differential dead time (van der Klis 1989), assuming a paralyzable process (Zhang et al. 1995) with a dead time of $10 \mu \mathrm{s}$ (Zhang 1995), ranged between $0.9 \%$ and $1.2 \%$ in observation 1 and $0.6 \%$ and $0.9 \%$ in observation 2. There was no clear dependence on $\dot{M}$ within each observation.

QPOs with a frequency of $\sim 800 \mathrm{~Hz}$ were observed simultaneously with the $1100 \mathrm{~Hz}$ QPOs (Fig. 2, top) only when the source was in the upper NB $\left(S_{Z}<1.36\right)$. From studying the short-term power spectral variations, we can exclude that the two peaks are due to one peak moving in frequency, unless it moves on timescales shorter than $32 \mathrm{~s}$. In orbit 2 of observation 1 , the QPO frequency $\nu_{800}$ generally increased (from $\sim 800$ to 
TABLE 1

QPO PARAMETERS

\begin{tabular}{|c|c|c|c|c|c|c|}
\hline \multirow[b]{2}{*}{$S_{Z}$} & \multicolumn{2}{|c|}{$1100 \mathrm{~Hz}$} & \multicolumn{2}{|c|}{$800 \mathrm{~Hz}$} & \multicolumn{2}{|c|}{ N/FBO } \\
\hline & $\begin{array}{l}\text { Frequency } \\
(\mathrm{Hz})\end{array}$ & $\begin{array}{c}\text { FWHM } \\
(\mathrm{Hz})\end{array}$ & $\begin{array}{l}\text { Frequency } \\
(\mathrm{Hz})\end{array}$ & $\begin{array}{c}\text { FWHM } \\
(\mathrm{Hz})\end{array}$ & $\begin{array}{l}\text { Frequency } \\
(\mathrm{Hz})\end{array}$ & $\begin{array}{c}\text { FWHM } \\
(\mathrm{Hz})\end{array}$ \\
\hline \multicolumn{7}{|c|}{ Observation 1 , Orbit 2} \\
\hline $1.252 \pm 0.027 \ldots$ & $1050.3 \pm 1.1$ & $41 \pm 5$ & $803 \pm 3$ & $88 \pm 11$ & $4.9 \pm 0.6$ & $13 \pm 3$ \\
\hline $1.282 \pm 0.040 \ldots$ & $1069.8 \pm 8.2$ & $104 \pm 30$ & $794 \pm 20$ & $145_{-50}^{+189}$ & $6.06 \pm 0.25$ & $9.3 \pm 1.1$ \\
\hline $1.336 \pm 0.032 \ldots$ & $1080.0 \pm 4.8$ & $50 \pm 19$ & $833 \pm 12$ & 80 fixed & $5.74 \pm 0.28$ & $10.7 \pm 1.1$ \\
\hline $1.340 \pm 0.050 \ldots \ldots \ldots$ & $1072.5 \pm 5.4$ & $78 \pm 18$ & $829 \pm 5$ & $49 \pm 19$ & $6.06 \pm 0.22$ & $8.5 \pm 0.8$ \\
\hline $1.357 \pm 0.022 \ldots \ldots \ldots$ & $1069 \pm 15$ & $178_{-65}^{+149}$ & $820 \pm 12$ & 80 fixed & $5.64 \pm 0.27$ & $9.9 \pm 1.1$ \\
\hline \multicolumn{7}{|c|}{ Observation 2 , Orbit 2} \\
\hline $1.866 \pm 0.022 \ldots \ldots \ldots$ & $1091.4 \pm 1.8$ & $22 \pm 5$ & $\ldots$ & $\ldots$ & $7.85 \pm 0.04$ & $4.44 \pm 0.12$ \\
\hline $1.929 \pm 0.050 \ldots$ & $1100.8 \pm 2.0$ & $18 \pm 4$ & $\cdots$ & $\cdots$ & $9.42 \pm 0.07$ & $7.24 \pm 0.23$ \\
\hline \multicolumn{7}{|c|}{ Observation 2 , Orbit 1} \\
\hline $1.966 \pm 0.032 \ldots \ldots \ldots$ & $1090.1 \pm 2.3$ & $18 \pm 6$ & $\ldots$ & $\ldots$ & $7.66 \pm 0.09$ & $5.81 \pm 0.27$ \\
\hline $2.057 \pm 0.006 \ldots \ldots \ldots$ & $1129.7 \pm 2.3$ & $13.4_{-1.4}^{+6.2}$ & $\cdots$ & $\ldots$ & $13.64 \pm 0.17$ & $6.6 \pm 0.6$ \\
\hline $2.092 \pm 0.025 \ldots \ldots \ldots$ & $1135 \pm 13$ & $68_{-40}^{+88}$ & $\ldots$ & $\ldots$ & $13.46 \pm 0.04$ & $9.60 \pm 0.18$ \\
\hline
\end{tabular}

NOTES.-Fitting function: see $\S 3$. All errors correspond to unreduced $\Delta \chi^{2}=1$. Fit range, frequency resolution, and number of degrees of freedom for the 1100 and $800 \mathrm{~Hz}$ QPO fits were $200-2000 \mathrm{~Hz}, 10 \mathrm{~Hz}$, and 171 for observation 1, orbit 2; 512-1536 Hz, $5 \mathrm{~Hz}$, and 200 for observation 2, orbit 1; and $200-2000 \mathrm{~Hz}, 5 \mathrm{~Hz}$, and 356 for observation 2 , orbit 2 , respectively.

$\sim 830 \mathrm{~Hz}$ ) when $S_{Z}$ rose from 1.25 to $1.35\left(\nu_{1100}\right.$ increased from 1050 to $1075 \mathrm{~Hz}$ over the same range). The $800 \mathrm{~Hz}$ QPOs were relatively broad, from $50 \mathrm{~Hz}$ to greater than $100 \mathrm{~Hz}$, and had amplitudes between $0.9 \%$ and $1.2 \%$ (rms), decreasing slightly with $S_{Z}$. In orbit 1 of observation $1\left(S_{Z}<1.16\right)$, the sensitivity was less due to the lack of double-event data, but there was some evidence $(3 \sigma)$ for the presence of a broad peak near $720 \mathrm{~Hz}$.

A third new QPO feature, located near $45 \mathrm{~Hz}$, was detected on the NB. These QPOs had an FWHM of typically $15-25 \mathrm{~Hz}$ and an amplitude of $\sim 1 \%(\mathrm{rms})$ and were most prominent on the middle of the NB, near $S_{Z}=1.5$. They were detected all the way from $S_{Z}=1.1$ to 1.9 with little change in frequency. Figure 3 shows the power spectrum of data obtained in orbit 3 of observation 1, with $6 \mathrm{~Hz}$ QPOs (the N/FBOs) and $45 \mathrm{~Hz}$ QPOs.

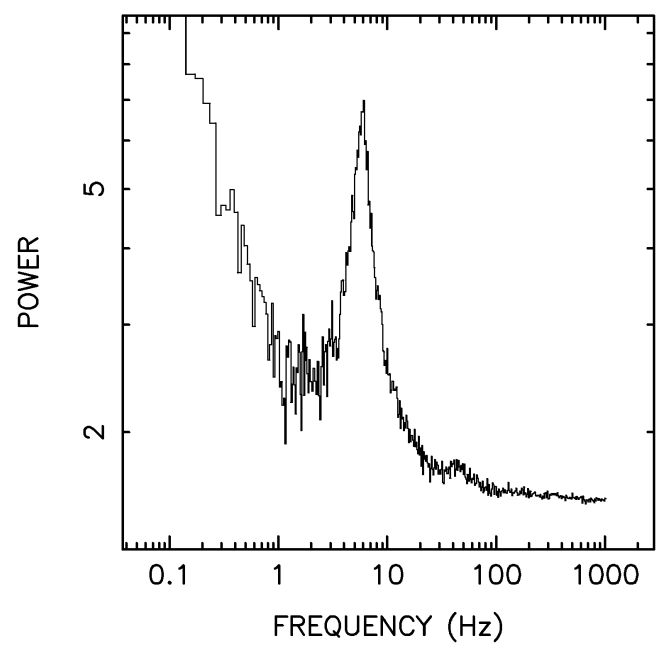

FIG. 3.-Power spectrum of $0.5 \mathrm{~ms}$ resolution data obtained in orbit 3 of observation 1, showing a strong N/FBO peak centered on $6 \mathrm{~Hz}$ and a weaker feature near $45 \mathrm{~Hz}$. The spectrum is Leahy-normalized; the offset of the continuum from a level of 2.0 is due to dead-time effects.
The dependencies of $\nu_{1100}$ and the N/FBO frequency $\nu_{\mathrm{N} / \mathrm{FBO}}$ on $S_{Z}$ are very similar. The relation between $\nu_{1100}$ and $\nu_{\mathrm{N} / \mathrm{FBO}}$ is plotted in Figure 4. There is a strong correlation. The relation fits a straight line, with $\nu_{1100}=7.3 \nu_{\mathrm{N} / \mathrm{FBO}}+1032$; a power law fits as well.

\section{DISCUSSION}

The discovery of near-millisecond (Strohmayer, Zhang, \& Swank 1996b; van Paradijs et al. 1996) and submillisecond (van der Klis et al. 1996) oscillations from accreting low magnetic field neutron stars was expected on the basis of general arguments (see § 1). To find and study such phenomena was one of the primary objectives of the RXTE mission. There is a range of phenomena that could take place on these timescales, and we shall not attempt to be exhaustive here. The

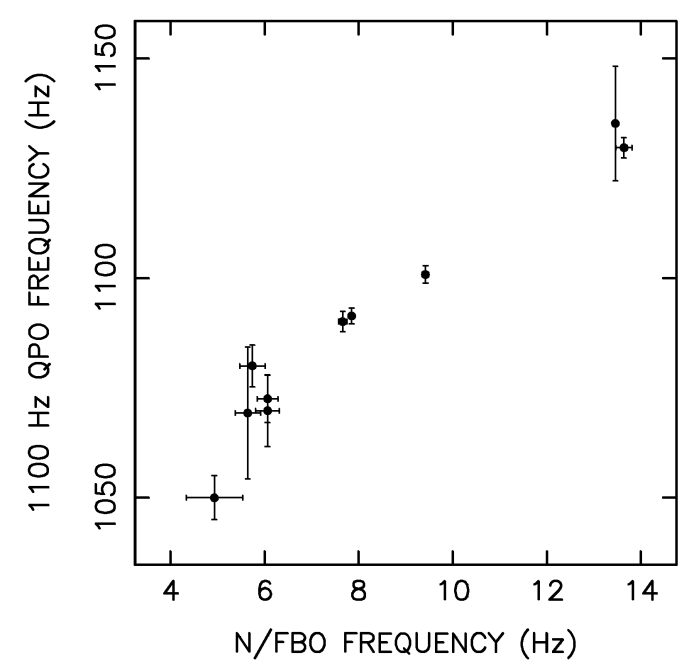

FIG. 4.- Relation between the frequencies of the $1100 \mathrm{~Hz}$ QPOs and the N/FBOs as derived from data in which these two phenomena were detected simultaneously. A strong correlation is evident. 
fact that both $\nu_{1100}$ and $\nu_{800}$ increase considerably as a function of $\dot{M}$ suggests an origin in accretion phenomena rather than neutron star vibrations (e.g., McDermott, Van Horn, \& Hansen 1988). Therefore, we concentrate on that class of models.

Disk frequency. - The Kepler frequency $\nu_{\mathrm{K}}$ at the inner edge of the accretion disk, bounded by a small $(16-20 \mathrm{~km})$ magnetosphere, is in the correct range to be identified with either $\nu_{1100}$ or $\nu_{800}$. It would increase with $\dot{M}$, as observed. The $\dot{M}$-dependence of the $800 \mathrm{~Hz}$ QPOs, strongest at low $\dot{M}$ and disappearing toward higher $\dot{M}$, is reminiscent of the "horizontal-branch QPOs" (HBOs) in other Z sources (van der Klis et al. 1985; see van der Klis 1995 for a review). These HBOs, in the beat-frequency model (Alpar \& Shaham 1985; Lamb et al. 1985), are caused by "clumps" in the inner disk, which are most prominent at the lowest $\dot{M}$-levels. The $800 \mathrm{~Hz}$ QPOs (or, alternatively, the $1100 \mathrm{~Hz}$ QPOs) could be a direct signal from these clumps, either by reflection or obscuration of the X-ray flux from the central regions or by interaction with azimuthal structure in the radial flow (see below).

Beat frequency.-As no HBOs have been detected as yet from Sco X-1, an alternative possibility is that the $800 \mathrm{~Hz}$ QPOs are HBOs and that their frequency is the beat frequency $\nu_{B}=n\left(\nu_{\mathrm{K}}-\nu_{s}\right)$ between $\nu_{\mathrm{K}}$ and the neutron star spin frequency $\nu_{s}$, where $n$ is the field symmetry factor. This would make the HBOs in Sco X-1 much faster than those in other Z sources, whose frequency is $15-55 \mathrm{~Hz}$, and would require a relatively slow neutron star spin rate (not more than a few hundred hertz). When this Letter was about to be submitted, the detection was reported in 4U 1728-34 of $363 \mathrm{~Hz}$ oscillations during X-ray bursts (Strohmayer et al. 1996a), which were interpreted as being due to the neutron star spin, and of two variable-frequency QPO peaks (one of which is the $\sim 800 \mathrm{~Hz}$ QPO reported by Strohmayer et al. 1996b) whose separation remained near $363 \mathrm{~Hz}$. Clearly, this suggests a beat-frequency model interpretation with $\nu_{B}, \nu_{\mathrm{K}}$, and $\nu_{s}$ all visible. In the case of Sco X-1, the difference $\nu_{1100}-\nu_{800}$ is consistent with being constant near $250 \mathrm{~Hz}$, but the range in frequencies over which both peaks are detected simultaneously is too small to draw any strong conclusion from this; there is no evidence for an oscillation near $250 \mathrm{~Hz}$.

The new $45 \mathrm{~Hz}$ QPOs in Sco X-1 are in the range of HBO frequencies seen in the other $\mathrm{Z}$ sources. The dependence of their amplitude on $S_{Z}$ is clearly different from that of HBOs in GX 5-1 and Cyg X-2, which are strongest at the top of the NB (and in the $\mathrm{HB}$ ), not in the middle of the NB. In GX 17+2, $60 \mathrm{~Hz}$ QPOs have been discovered in the NB, which may be a similar phenomenon (Wijnands et al. 1996). If the $45 \mathrm{~Hz}$ QPOs in Sco X-1 are beat-frequency QPOs, then from the fact that its frequency is approximately constant we conclude that the 1100 and $800 \mathrm{~Hz}$ QPOs, whose frequencies vary by tens of hertz, cannot be the disk frequency.

Doppler-shifted pulsar. - The strong correlation we observe between $\nu_{1100}$ and $\nu_{\mathrm{N} / \mathrm{FBO}}$ is not explained by any of the models discussed above. It could in principle arise because both frequencies vary as a function of $\dot{M}$ independently of each other, but this seems unlikely. The $\nu_{\mathrm{N} / \mathrm{FBO}}$ versus $S_{Z}$ relation is known to jump at $S_{Z}=2$ (see, e.g., van der Klis 1995), and $\nu_{1100}$ should then, independently, show a similar jump in its dependence on $S_{Z}$ to maintain the correlation. It seems more likely that one frequency is by some mechanism directly derived from the other.

We briefly explore a mechanism in which this is the case. In the Fortner, Lamb, \& Miller (1989) model for N/FBOs, part of the accretion takes place by way of an approximately radial inflow, and blobs (inhomogeneities) in this flow produce the $\mathrm{N} / \mathrm{FBOs}$. For a constant-size radial flow, $\nu_{\mathrm{N} / \mathrm{FBO}}$ is proportional to the inflow velocity $v_{r}$. If the $1100 \mathrm{~Hz}$ QPOs are the Doppler-shifted pulsar signal, which we see reflected off blobs in the flow, then when $v_{r}$ varies, $\nu_{1100}$ should vary linearly with $\nu_{\mathrm{N} / \mathrm{FBO}}$, as observed. For a blob moving toward the star along a radial vector that makes an angle $\phi$ with the line of sight, $\nu_{1100}$ should be proportional to $\left[1+(1-\cos \phi) v_{r} / c\right] \nu_{\text {puls }}$, where $\nu_{\text {puls }}$ is the pulse frequency. Radial flow velocities up to at least $0.04 c$ and spin frequencies of $\sim 500$ or $\sim 1000 \mathrm{~Hz}$, depending on whether one or two magnetic poles contribute to the signal, are required for this model to work. To obtain the relatively coherent QPO signal we observe, the range of angles $\phi$ contributing to the signal should be small. This would be true, for example, if the magnetic and rotation axes were nearly aligned. Aligned rotation would also explain why millisecond pulsars in low-mass X-ray binaries have been hard to find. This model would require a spin period $\sim 1 \mathrm{~ms}$, faster than any neutron star spin yet measured, as only one pole would contribute (the other pole could produce another QPO peak).

Note added in manuscript.-Further RXTE observations of Sco X-1 on May 24-25 again show the two high-frequency QPO peaks, this time with frequencies near 900 and $600 \mathrm{~Hz}$. Their separation is significantly $(>10 \sigma)$ larger than in the February observations. This excludes the beat-frequency interpretation in which the peak separation is predicted to be constant at the neutron star spin frequency.

This work was supported in part by the Netherlands Organization for Scientific Research (NWO) under grant PGS 78-277 and by the Netherlands Foundation for Research in Astronomy (ASTRON) under grant 781-76-017. W. H. G. L. and J. v. P. acknowledge support from the National Aeronautics and Space Administration.

\section{REFERENCES}

Alpar, M. A., \& Shaham, J. 1985, Nature, 316, 239

Bradt, H. V., Rothschild, R. E., \& Swank, J. H. 1993, A\&AS, 97, 355

Dieters, S., \& van der Klis, M. 1996, A\&A, submitted

Fortner, B. F., Lamb, F. K., \& Miller, G. S. 1989, Nature, 342, 775

Giacconi, R., Gursky, H., Paolini, F., \& Rossi, B. 1962, Phys. Rev. Lett., 9, 439

Hasinger, G., \& van der Klis, M. 1989, A\&A, 225, 79

Hasinger, G., van der Klis, M., Ebisawa, K., Dotani, T., \& Mitsuda, K. 1990, $\mathrm{A} \& \mathrm{~A}, 235,131$

Hertz, P., Vaughan, B., Wood, K. S., Norris, J. P., Mitsuda, K., Michelson, P. F. \& Dotani, T. 1992, ApJ, 396, 201

Lamb, F. K. 1991, in Neutron Stars: Theory and Observation, ed. J. Ventura \& D. Pines (NATO ASI Ser. C, 344) (Dordrecht: Kluwer), 445

Lamb, F. K., Shibazaki, N., Alpar, M. A., \& Shaham, J. 1985, Nature, 317, 681

McDermott, P. N., Van Horn, H. M., \& Hansen, C. J. 1988, ApJ, 325, 725

Middleditch, J., \& Priedhorsky, W. C. 1986, ApJ, 306, 230

Priedhorsky, W. C., Hasinger, G., Lewin, W. H. G., Middleditch, J., Parmar, A.

Stella, L., \& White, N. 1986, ApJ, 306, L91
Strohmayer, T., Zhang, W., Smale, A., Day, C., Swank, J., Titarchuk, L., \& Lee, U. 1996a, IAU Circ. 6387

Strohmayer, T., Zhang, W., \& Swank, J. 1996b, IAU Circ. 6320

van der Klis, M. 1989, in Timing Neutron Stars, ed. H. Ögelman \& E. P. J. van den Heuvel (NATO ASI Ser. C, 262) (Dordrecht: Kluwer), 27 . 1995, in X-Ray Binaries, ed. W. H. G. Lewin, J. van Paradijs, \& E. P. J van den Heuvel (Cambridge: Cambridge Univ. Press), 252

van der Klis, M., Jansen, F., van Paradijs, J., Lewin, W. H. G., van den Heuvel, E. P. J., Trümper, J. E., \& Sztajno, M. 1985, Nature, 316, 225

van der Klis, M., Swank, J., Zhang, W., Jahoda, K., Morgan, E., Lewin, W. Vaughan, B., \& van Paradijs, J. 1996, IAU Circ. 6319

van Paradijs, J., et al. 1996, IAU Circ. 6336

Wijnands, R. A. D., van der Klis, M., Psaltis, D., Lamb, F. K., Kuulkers, E. Dieters, S., van Paradijs, J., \& Lewin, W. H. G. 1996, ApJ, 469, L5 Zhang, W. 1995, XTE/PCA internal memo.

Zhang, W., Jahoda, K., Swank, J. H., Morgan, E. H., \& Giles, A. B. 1995, ApJ 449, 930 